\title{
Concepcão de um sistema info-eletrônico de saúde integrado a um aplicativo de notificações para clínicas odontológicas
}

\author{
Design of an info-electronic health system integrated to a notification application for dental clinics \\ Concepción de un sistema de salud infoelectrónico integrado con una aplicación de notificación \\ para clínicas dentales
}

Recebido: 15/11/2021 | Revisado: 24/11/2021 | Aceito: 26/11/2021 | Publicado: 07/12/2021

Bruno Jean Santos Bomfim
ORCID: https://orcid.org/0000-0001-6657-7038
Faculdade Independente do Nordeste, Brasil
E-mail: brunojeean @ gmail.com
Gustavo Alves Oliveira
ORCID: https://orcid.org/0000-0001-8051-0462
Faculdade Independente do Nordeste, Brasil
E-mail: oliveira.acad21@gmail.com
Patrícia Maria Coelho
ORCID: https://orcid.org/0000-0003-1319-4441
Faculdade Independente do Nordeste, Brasil
E-mail:patriciamacoelho@ gmail.com

\begin{abstract}
Resumo
Uma grande parte da atividade dos profissionais de saúde consiste em processar informações, ou seja, a obtenção e registro dos casos clínicos, informações sobre o paciente, seja por questões legais ou a título de comparação inicial e final do tratamento proposto ao paciente. Levando essa realidade em consideração, em que todas estas atividades demandam muito tempo e uma estrutura especifica, o presente artigo tem como objetivo descrever um sistema de rede de informação e um aplicativo para dispositivos móveis, com elementos próprios a essa nova tecnologia, aplicáveis tanto a profissionais da saúde como aos pacientes, recursos como: acessibilidade, mobilidade e habilidade continua de transferência de dados em tempo real. O protótipo foi construído de forma estruturada, a partir de um projeto piloto previamente testado para a escolha assertiva do desenvolvimento de conteúdo, escolha do software de criação, formatação, layout e indexação em lojas de aplicativos. Após o protótipo do aplicativo ter sido testado com êxito, permitiu-se passar as etapas seguintes, incluindo-se a testagem pelos profissionais e pacientes o aplicativo desenvolvido demonstrou ser uma ferramenta de auxílio ao diagnóstico baseado em evidências e literatura científica para complementar e orientar o profissional cirurgião-dentista acerca da prevenção, diagnóstico e tratamento de diversas patologias orais, além de orientar pacientes quanto a cuidados, e à sua agenda. Sendo assim, poderá após finalizado, o aplicativo poderá ser indexado as lojas de aplicativos (App Store e Google Play), com custo para download, além de necessário o cadastro para a utilização.
\end{abstract}

Palavras-chave: TIC na saúde; Aplicativos móveis; Competência em informação; Informática odontológica.

\begin{abstract}
A large part of the activity of health professionals consists of processing information that is, obtaining and recording clinical cases, information about the patient, either for legal reasons or as an initial and final comparison of the treatment proposed to the patient. Taking this reality into account, in which all these activities demand a lot of time and a specific structure, this article aims to describe an information network system and an application for mobile devices, with elements specific to this new technology, applicable both to healthcare professionals like to patients, features such as: accessibility, mobility and continuous ability to transfer data in real time. The prototype was built in a structured way, from a pilot project previously tested for the assertive choice of content development, choice of creation software, formatting, layout and indexing in app stores. After the prototype of the application was successfully tested, the following steps were allowed to pass, including testing by professionals and patients. The developed application proved to be a tool to aid diagnosis based on evidence and scientific literature to complement and guide the dental surgeon about the prevention, diagnosis and treatment of various oral pathologies, in addition to advising patients about care and their agenda. Thus, after finalized, the application can be indexed in the application stores (App Store and Google Play), with a cost for downloading, in addition to being necessary to register for use.
\end{abstract}

Keywords: ICT in health; Mobile apps; Information literacy; Dental informatics. 


\begin{abstract}
Resumen
Gran parte de la actividad de los profesionales de la salud consiste en procesar información, es decir, obtener y registrar casos clínicos, información sobre el paciente, ya sea por motivos legales o como comparación inicial y final del tratamiento propuesto al paciente. Teniendo en cuenta esta realidad, en la que todas estas actividades demandan mucho tiempo y una estructura específica, este artículo pretende describir un sistema de red de información y una aplicación para dispositivos móviles, con elementos propios de esta nueva tecnología, aplicable tanto a los profesionales sanitarios gusta a los pacientes, características como: accesibilidad, movilidad y capacidad continua para transferir datos en tiempo real. El prototipo se construyó de forma estructurada, a partir de un proyecto piloto previamente probado para la elección asertiva del desarrollo de contenido, la elección del software de creación, el formato, el diseño y la indexación en las tiendas de aplicaciones. Una vez que se probó con éxito el prototipo de la aplicación, se permitió que pasaran los siguientes pasos, incluidas las pruebas realizadas por profesionales y pacientes. La aplicación desarrollada resultó ser una herramienta de ayuda al diagnóstico basado en la evidencia y la literatura científica para complementar y orientar al cirujano dentista en la prevención, diagnóstico y tratamiento de diversas patologías bucales, además de asesorar a los pacientes sobre los cuidados y su agenda. Así, una vez finalizada, la aplicación se puede indexar en las tiendas de aplicaciones (App Store y Google Play), con un costo de descarga, además de ser necesario registrarse para su uso.
\end{abstract}

Palabras clave: TIC en la salud; Aplicaciones móviles; Alfabetización informacional; Informática dental.

\title{
1. Introdução
}

As Tecnologias Info-Eletrônicas voltadas para a área da saúde, detêm diversas ferramentas e recursos que contribuem para uma melhor composição, e organização de dados e informações. São programas de software que funcionam em computadores, tablets, smartphones e outros dispositivos móveis. Proporcionando, assim, um seguro armazenamento, acesso e compartilhamento de informações, seja entre os profissionais da área envolvidos no tratamento, ou pelo próprio paciente (Guimarães \& Godoy, 2016; Barra et al., 2017).

A construção desse Sistema Info-Eletrônico deverá viabilizar a comunicação, transmissão e inovação das informações na área da saúde, e assim auxiliar e facilitar o processo de diagnóstico, tomada de decisões clínicas, e condutas terapêuticas que serão realizadas no tratamento dos pacientes (Santos et al., 2016; Mendes, 2011). Outro atributo favorável é a mobilidade, o sistema, permitirá o acesso em qualquer hora, em qualquer lugar, fora e/ou dentro do ambiente físico do consultório. Isso proporcionará uma maior autonomia no alcance das informações, sendo possível traçar qual tipo de procedimento fazer de acordo com o tempo disponível, promovendo uma ampla cobertura da assistência à saúde (Martin, et al., 2016; Ribeiro, et al., 2020).

O aplicativo, será uma ferramenta que poderá ser utilizada para estimular o autocuidado, nesse sentido, tende a aumentar o comprometimento e o interesse dos pacientes com sua própria saúde, já que facilitará a transmissão de informações, os motivando a uma rotina de higiene oral. O aplicativo facultará que os usuários definam lembretes de escovação dental pelo menos três vezes ao dia, uso do fio dental, uso de enxaguantes bucais, avisos quanto a próxima consulta com o seu dentista e troca da escova de dentes a cada três meses. E a utilização de diversos recursos de mídias, tais como: imagens, vídeos, sons e movimentos, como os atlas de anatomia, jogos que podem ser facilmente inseridos em condutas terapêuticas e de cuidados à saúde (Free, et al., 2010). Além de poder oferecer um suporte, de consulta online através do app, para tirar dúvidas ou atendimento de emergências com a equipe de saúde, sem precisar passar o número de contato pessoal do cirurgião dentista.

O uso da tecnologia no contexto da saúde tornou-se adequado a partir de elementos que são intrínsecos à sua aplicabilidade, consentindo que as práticas de promoção à saúde possam ser desenvolvidas e transmitidas, facilitando a integração entre equipe multiprofissional e usuário/paciente. Essa funcionalidade tornou-se possível pelo progresso da tecnologia da informação ao ciberespaço global, associado ao surgimento e inovação dos aplicativos (Apps) para celulares smartphones, tablets, notebooks e afins, que possuem, entre seus atributos, o fácil manejo intuitivo e o maior acesso à informação por profissionais e paciente, as quais podem favorecer o binômio prognóstico-autocuidado (Rocha, et al., 2017).

Até o momento, ainda não foi projetado nenhum aplicativo para dispositivos móveis com essas finalidades, ou seja, com a entrega de configurações referentes ao tratamento, prognóstico, e aos cuidados odontológicos. Com esse intuito, esse 
artigo irá relatar a criação de um sistema de rede de informação e aplicativo para dispositivos móveis, tendo entre seus objetivos tornar elementos que são próprios a essa nova tecnologia, aplicáveis, tanto a profissionais da saúde bem como aos pacientes, recursos como: acessibilidade, mobilidade e habilidade contínua de transferência de dados, inclusive, em tempo real.

\section{Materiais e Métodos}

Trata-se de um projeto que foi desenvolvido por uma equipe composta por um Cirurgião-Dentista e um estudante de odontologia, um técnico de informática e um designer gráfico. O protótipo foi construído de forma estruturada, determinada pelo projeto piloto que visou o desenvolvimento de conteúdo, escolha do software de criação, formatação, layout, indexação em lojas de aplicativos.

Foi realizado uma busca nas bases de dados PUBMED, SCIELO, LILACS, nos idiomas inglês e português, utilizando descritores específicos: ("Tecnologia da Informação"; "Aplicativos móveis"; "Competência informacional”; "Informática Odontológica" / "Information Technology"; "Mobile apps"; "Informational competence"; "Dental Informatics"), juntamente com o operador booleano "AND” de forma a combinar os descritores citados.

Foram incluídas informações de estudos atualizados, dando preferência para revisões sistemáticas e estudos clínicos randomizados.

O sistema foi construído por meio do softwarePhoneGap, e do framework (softs de desenvolvimento móvel). Como escolhas de plataformas de sistemas operacionais de smartphones serão incluídos no IOS e Android, contemplando grande parte do mercado disponível. A formatação e o layout do aplicativo estão sendo desenvolvidos para se ter uma maior acessibilidade, sendo providos com um menu inicial composto de "cadastro" (imprescindível para o uso), "profissionais" e "pacientes".

Após acessar o menu de acordo com a categoria, um submenu composto de "Prognóstico" - para ter acesso aos diagnósticos, planejamento e as estratégias de tratamento, resultados de laboratório e radiológicos e comprometimentos sistêmicos; "Cuidados" - para ter acesso às manifestações bucais e protocolos de cuidado odontológico; "Consultas" - para ter informações sobre os agendamentos das consultas; e "Financeiro" - controle de todo investimento feito ou a ser realizado.

\section{Resultados}

Após o teste realizado com o protótipo piloto, todas as funcionalidades do sistema apresentaram êxito em suas execuções. Permitiu, desta forma, passar às etapas seguintes, incluindo a testagem pelos profissionais e pacientes.

O aplicativo, assumiu desta maneira, o papel de mediador e facilitador entre estes avanços tecnológicos e seus usuários em potencial. Funcionou de maneira auxiliar na promoção dos cuidados à saúde, principalmente pelo maior acesso a informações, juntamente com a participação do usuário no seu tratamento.

Sendo assim, após finalizado, o aplicativo será indexado às lojas de aplicativos (App Store e Google Play), com custo para download, além de necessário o cadastro para a utilização.

\section{Discussão}

Com o advento da internet por meio dos dispositivos móveis surgiu uma subdivisão da saúde chamado de Saúde Móvel (mHealth). E de acordo a OMS (2011), embora não se tenha uma padronização, entende-se que seja a oferta de serviços médicos e/ou saúde pública que se faça a utilização de tecnologias disponíveis em dispositivos móveis, como smartphones, e sensores conectados ao usuário (Oliveira, et al., 2017).

Silva et al. (2016) relatam que a internet se tornou algo tão presente na vida das pessoas e no convívio social, trouxe facilidades e modificou vários contextos, incluindo na saúde. Já Castro et al. (2015) salientam que as TICs (Tecnologias da 
Informação e Comunicação) são ofertadas aos usuários dos serviços de internet, fazendo que assim se amplie o processo de construção, monitoramento e compartilhamento de dados. Outrossim, Jacopetti (2011) diz que se usada da forma correta as tecnologias digitais e os indivíduos podem ser de maneira igual agentes ativos no empenho de se buscar o conhecimento sobre sua própria saúde.

Segundo um estudo de Junior, D’Castro et al. (2011), na atualidade, mesmo após uma boa consulta com qualquer profissional de saúde, os pacientes tendem ao uso da internet para esclarecer dúvidas remanescentes e aprofundar seus conhecimentos sobre sua patologia e o processo de saúde-doença. Já Chaves et al. (2018); e Oliveira e Santos (2018) escrevem que há vários aplicativos voltados a categoria multiprofissional, que utilizam dados dos pacientes como conteúdo de estudo, monitorando os seus sinais vitais, mantendo assim uma comunicação com os membros daquela equipe de saúde. Em outros há a possibilidade do relato diário do paciente sobre o estágio da sua doença, como está se sentindo ao decorrer do dia, além de fazer anotações importantes sobre o seu estado.

Montiel et al. (2015), a partir das informações dispostas em seu estudo corroboraram no entendimento de que as TICs quando ligadas à saúde tem a possibilidade de contribuir de forma significativa na redução dos índices de erros clínicos, na melhoria dos cuidados em saúde e aumentar a eficiência e qualidade de vida do paciente.

Rocha et al., (2017) relatam que o uso de tecnologias com o intuito de monitorar, promover cuidados e uma maior adesão aos tratamentos de saúde facilitam a integração entre profissional-paciente. Ainda pode ser observado que há um fluxo contínuo que é permeado pela troca de informações constantemente entre os envolvidos nesses processos. E isso só foi possível pelo avanço do cyber space, e doas aplicativos para telefones móveis (Apps), e que tem, entre suas características, a facilidade de utilização e um maior acesso à informação.

Tiffany et al. (2018) fizeram um levantamento de aplicativos disponíveis relacionados a orientações de higiene oral em alguns aspectos: bases teóricas ou empíricas, características descritivas, funcionalidades e se eram instruídos quanto a fatores causais de doenças do sistema estomatognático, como dieta, etilismo e tabagismo. E a conclusão foi de que nenhum ofereceu adequado embasamento teórico, e de modo geral, estes apps oferecem ferramentas que possibilitam monitorar ou fazer lembretes de escovação e assistências em marcações de consultas.

De acordo com o estudo de Bohn et al. (2018), a visão que os pacientes tem é que os aplicativos destinados a orientações e instruções de saúde e higiene oral tem que ser usados sob orientação e controle de um Cirurgião-Dentista. A vontade deles é que os apps possam ser personalizados para cada paciente. E ainda relataram preferir características interativas e imagens mais lúdicas.

O estudo de Cozzani et al. (2016) investigou o efeito que telefonemas e mensagens de texto podem ter sobre a condescendência dos pacientes com as instruções de higiene oral, em grupo controle. Verificou-se que os grupos os quais receberam estas intervenções (ligação ou mensagem) apresentaram menor índice de placa. Outros estudos de Alkadhi et al. (2017); Kumar et al. (2018) mostraram que lembretes feitos por meios eletrônicos uma a três vezes por semana para os pacientes ou aos seus pais se mostrou eficaz na melhoria da higiene oral nesses pacientes. E, segundo Abdaljawwad (2016) relatou que a introdução de mensagens de texto tornou mais eficaz a comunicação com o paciente.

\section{Considerações Finais}

Por vivermos em uma sociedade que necessita ser suprida por informação e conhecimento, as tecnologias digitais vêm dar o suporte para que as céleres mudanças estejam disponíveis em tempo oportuno e real. E por conta da facilidade da tecnologia móvel e de diversos recursos educacionais, existe a oportunidade que todos os indivíduos do mundo tenham a acesso à educação digital. Logo, a educação em saúde é beneficiada neste contexto, partindo do pressuposto que a comunicação entre os profissionais e os pacientes é favorecida. 
O aplicativo desenvolvido mostrou ser uma ferramenta de auxílio no diagnóstico e arquivo de documentação legalmente aceita, pois consegue arquivar todos os dados e exames complementares necessários do paciente. Permite aos pacientes ter acesso ao seu planejamento, plano financeiro e cuidados com sua saúde oral. Aumenta a interação paciente-profissional de maneira rápida e acessível.

Contudo, ainda há a necessidade de mais estudos para evidenciar a relevância do uso de aplicativos em dispositivos móveis no processo de educação em saúde bucal para a população em geral.

\section{Referências}

Abdaljawwad, A. A. (2016). The influence of text message reminders on oral hygiene compliance in orthodontic patients. Iraqi Dental Journal, 38(1), 58-62. http://www.iraqidentaljournal.com/index.php/idj/article/view/74/49

Alkadhi, O. H., Zahid, M. N., Almanea, R. S., Althaqeb, H. K., Alharbi, T. H., \& Ajwa, N. M. (2017). The effect of using mobile applications for improving oral hygiene in patients with orthodontic fixed appliances: a randomised controlled trial. Journal of orthodontics, 44(3), 157-163. https://doi.org/10.1080\%2F14653125.2017.1346746

Barra, D. C. C., Paim, S. M. S., Sasso, G. T. M. D., \& Colla, G. W. (2018). Métodos para desenvolvimento de aplicativos móveis em saúde: revisão integrativa da literatura. Texto \& Contexto-Enfermagem, 26 (4), 1-12. https://doi.org/10.1590/0104-07072017002260017

Bohn, C. E., McQuistan, M. R., McKernan, S. C., \& Askelson, N. M. (2018). Preferences related to the use of mobile apps as dental patient educational aids: A pilot study. Journal of prosthodontics, 27(4), 329-334. https://doi.org/10.1111/jopr.12667

Castro, E., Reis, E., Spinillo, C. G., \& de Oliveira, A. E. F. (2015). Interface gráfico-informacional de aplicativos de educação em saúde: uma análise do appSaúde da Criança I da UNA-SUS/UFMA. Blucher Design Proceedings, 2(2), 281-293. DOI 10.5151/designpro-CIDI2015-cidi_105

Chaves, A. S. C., Oliveira, G. M., de Jesus, L. M. D. S., Martins, J. L., \& da Silva, V. C. (2018). Uso de aplicativos para dispositivos móveis no processo de educação em saúde. Humanidades \& Inovação, 5(6), 34-42. https://revista.unitins.br/index.php/humanidadeseinovacao/article/view/744

Cozzani, M., Ragazzini, G., Delucchi, A., Mutinelli, S., Barreca, C., Rinchuse, D. J., \& Piras, V. (2016). Oral hygiene compliance in orthodontic patients: a randomized controlled study on the effects of a post-treatment communication. Progress in orthodontics, 17(1), 1-6. https://doi.org/10.1186/s40510-016-0154-

Free, C., Phillips, G., Felix, L., Galli, L., Patel, V., \& Edwards, P. (2010). The effectiveness of M-health technologies for improving health and health services: a systematic review protocol. BMC research notes, 3(1), 1-7. https://doi.org/10.1186/1756-0500-3-250

Guimarães, E. M. P., \& Godoy, S. C. B. (2012). Telenfermagem-Recurso para assistência e educação em enfermagem. Rev Min Enferm, 16(2), 157-8. http://www.reme.org.br/artigo/detalhes/513

Jacopetti, A. (2011). Práticas sociais e de comunicação de pacientes renais no Facebook da Fundação Pró-Rim. Revista de Estudos da Comunicação, 12(27), 81-89. https://doi.org/10.7213/rec.v12i27.22359

Junior, J. V. M., D'Castro, R. J., Rodrigues, F. M. M., de Gusmão, C. M. G., Lyra, N. R. S., \& Sarinho, S. W. (2011). InteliMed: uma experiência de desenvolvimento de sistema móvel de suporte ao diagnóstico médico. Revista Brasileira de Computação Aplicada,3(1), 30-42. https://doi.org/10.5335/rbca.2013.1316

Kumar, G. S., Kashyap, A., Raghav, S., Bhardwaj, R., Singh, A., \& Guram, G. (2018). Role of text message reminder on oral hygiene maintenance of orthodontic patients. J Contemp Dent Pract, 19(1), 98-101. https://www.thejcdp.com/doi/pdf/10.5005/jp-journals-10024-2219

Martin, C. K., Gilmore, L. A., Apolzan, J. W., Myers, C. A., Thomas, D. M., \& Redman, L. M. (2016). Smartloss: a personalized mobile health intervention for weight management and health promotion. JMIR mHealth and uHealth, 4(1), e5027. https://doi.org/10.2196/mhealth.5027

Mendes, E. V. (2011). As redes de atenção à saúde. Organização Pan-Americana da Saúde.

Montiel, E., Baldi, A., Kluthcovsky, A., \& Castro Jr, A. (2015, October). Sistema de Educação em Saúde no Tratamento do Diabetes Mellitus Tipo 2. In Brazilian Symposium on Computers in Education (Simpósio Brasileiro de Informática na Educação-SBIE) 26(1), 328. http://dx.doi.org/10.5753/cbie.sbie.2015.328

Oliveira, G. M., \& Santos, L. F. (2018). Uso de aplicativos para dispositivos móveis no processo de educação em saúde: reflexos da contemporaneidade. Revista Observatório, 4(6), 826-844. https://doi.org/10.20873/uft.2447-4266.2018v4n6p826

Oliveira, R. A. D., Lucena, T. F. R., \& Bernuci, M. P. (2017). Empoderamento em saúde bucal: M-Health no controle de cárie precoce da primeira infância (CPI). http://rdu.unicesumar.edu.br/handle/123456789/1899

Ribeiro, I. S. S., Rodrigues, B. T. G., Campos, A. R., de Menezes Maciel, R., \& Israel, M. S. (2020). Aplicativo Bucal App Como Ferramenta No Diagnóstico De Lesões Bucais: Revisão De Literatura. Ciência Atual-Revista Científica Multidisciplinar do Centro Universitário São José, 16(2). https://revista.saojose.br/index.php/cafsj/article/view/462

Rocha, F. S., Santana, E. B., da Silva, É. S., Carvalho, J. S. M., \& Carvalho, F. L. Q. (2017). Uso de Apps para a promoção dos cuidados à saúde. Anais do Seminário Tecnologias Aplicadas a Educação e Saúde. https://www.revistas.uneb.br/index.php/staes/article/view/3832 
Research, Society and Development, v. 10, n. 16, e83101623351, 2021

(CC BY 4.0) | ISSN 2525-3409 | DOI: http://dx.doi.org/10.33448/rsd-v10i16.23351

Santos, Z. M. S. A., Frota, M. A., \& Martins, A. B. T. (2016). Tecnologias em saúde: da abordagem teórica a construção e aplicação no cenário do cuidado. Fortaleza: EdUECE. http://www.uece.br/eduece/dmdocuments/Ebook\%20-\%20Tecnologia\%20em\%20Saude\%20-\%20EBOOK.pdf

Silva, B., Neto, F. M., Maia, C., \& Dutra, J. (2016, November). Um Ambiente Social Virtual de Auxílio à Aprendizagem Informal na Área de Saúde. In Anais dos Workshops do Congresso Brasileiro de Informática na Educação, 5 (1), 761-770. http://dx.doi.org/10.5753/cbie.wcbie.2016.761

Tiffany, B., Blasi, P., Catz, S. L., \& McClure, J. B. (2018). Mobile apps for oral health promotion: content review and heuristic usability analysis. JMIR mHealth and uHealth, 6(9), e11432. https://doi.org/10.2196/11432

WHO Global Observatory for eHealth. (2011). mHealth: new horizons for health through mobile technologies: second global survey on eHealth. World Health Organization. https://apps.who.int/iris/handle/10665/44607 